\title{
Real-time reconstruction of external impact on fractional order system under measuring a part of coordinates
}

\author{
P.G. Surkov* \\ Krasovskii Institute of Mathematics and Mechanics, 16 S. Kovalevskaya Str., Yekaterinburg, 620108, Russia \\ Yeltsin Ural Federal University, 19 Mira, Str., Yekaterinburg, 620002, Russia
}

\section{A R T I C L E I N F O}

\section{Article history:}

Received 17 February 2020

Received in revised form 29 May 2020

\section{MSC:}

34A08

$93 C 41$

$26 \mathrm{~A} 33$

49N45

Keywords:

Fractional order systems

Reconstruction

Input action

Incomplete information

Error estimation

\begin{abstract}
A B S T R A C T
For a system of nonlinear fractional differential equations, the problem of reconstructing an unknown external impact is considered. It is complicated by the fact that only a part of system's parameters is available for measuring. An algorithm for solving this problem is proposed, which is resistant to informational noises and computational errors, and is based on regularization methods and constructions of guaranteed control theory. A numerical example illustrating the operation of the algorithm is considered.
\end{abstract}

(c) 2020 Elsevier B.V. All rights reserved.

\section{Introduction}

We consider a dynamical system described by nonlinear fractional differential equations with the Caputo derivative of order $\gamma \in(0,1)$. The system operates on a finite time interval and undergoes unknown external impact, which can be interpreted as a disturbance. The trajectory generated by it is unknown in advance; only instantaneous restrictions on the disturbance are determined. At discrete, frequent enough time moments, a part of the coordinates of the phase vector of the system is measured with an error. The problem is to construct an algorithm for finding the unknown disturbance that is resistant to informational noises and computational errors.

The problem under discussion is naturally treated as the inverse problem of the dynamics of controlled systems [1,2]. A number of features are inherent in it. First, information about the state of the system is forthcoming during its operation, i.e. in real-time mode. This is motivated by the fact that a situation often arises in practice when the behavior of the object under study is a priori unknown. In a posteriori formulation, such problems were solved, for example, in [1,3]. One of the approaches to solving the problem of dynamical input reconstruction of the effect was proposed in [4-7]. In this case, a system of ordinary differential equations (ODE) was considered. This approach, in one of its statements, is based on the idea of local regularization by the smoothing functional method (Tikhonov regularization) of the Krasovskii extremal shift method. There are several modifications to the approach, depending on the regularization method used, for example, the residual method. In this paper, we use the smoothing functional method [1]. Following the chosen approach to solving the reconstruction problem, an auxiliary system (a model) is introduced. After that, the initial problem is reduced to the

\footnotetext{
* Correspondence to: Krasovskii Institute of Mathematics and Mechanics, 16 S. Kovalevskaya Str., Yekaterinburg, 620108, Russia.

E-mail address: spg@imm.uran.ru.
} 
control problem of the model according to the feedback principle. This approach was used by many authors to solve different problems of dynamical reconstruction (see, for example, [8-13] for ODEs). In most of these works, the entire phase vector of the system was accessible for observation; in this article, it is available to measure only a part of the coordinates (for ODEs, this case was studied in [8-10]). A feature of the problem under discussion is the extension of this approach to a new class of systems, namely, systems of fractional order. This work continues the research begun in [14] for fractional differential equations. The main difference from the mentioned work is that only a part of the coordinates are available for measuring whereas in [14] there is a complete information about the system. This feature leads to significantly different algorithms for solving the problems of reconstructing an unknown external impact in the previous and present paper.

Fractional calculus is currently undergoing the rapid development in both theoretical and applied research, see [15-21] and bibliography in them. Fractional derivatives are increasingly used in describing the properties of materials having a self-similar, porous structure, such as polymers [22]. An advantage over systems with derivatives of an integer order is revealed when modeling hereditary properties and memory $[16,18]$. Fractional derivatives are used in optimal control problems [23,24], in risk theory [25], in research related to medicine [26,27], in solving identification problems [28] and a source problem [29].

The remainder of this paper is organized as follows. Basic definitions and preliminary results are given in Section 2. Section 3 presents a rigorous statement of the problem for a fractional order system with the Caputo derivative. The description of the algorithm for solving this problem is suggested in Section 4. The substantiation of its convergence presented by Theorem 1 is also given there. Theorem 2, with an upper bound for the rate of convergence of the algorithm, is proved in Section 5. The numerical simulation illustrating the operation of the proposed algorithm by the example of a specific dynamical fractional order system is presented in Section 6. Finally, we make conclusions in Section 7.

\section{Preliminarities}

Before giving a statement of the problem considered in this article we recall some concepts from fractional calculus.

Definition 1 ([15, p. 42]). A fractional integral of order $\gamma$ of an arbitrary function $f \in L_{1}\left(T, R^{n}\right)$ with origin at a point $\sigma$ is defined by the formula

$$
\left[I^{\gamma} f\right](t)=\frac{1}{\Gamma(\gamma)} \int_{\sigma}^{t}(t-s)^{\gamma-1} f(s) d s, \quad \gamma \in(0,1), \quad t \in T=[\sigma, \theta], \quad \theta<+\infty,
$$

where $\Gamma(\cdot)$ is the Euler Gamma function [15, p. 29].

Definition 2. For a function $x: T \rightarrow R^{n}$ and an arbitrary real number $\gamma \in(0,1)$, the expression

$$
\left[D^{\gamma} x\right](t)=\frac{1}{\Gamma(1-\gamma)} \frac{d}{d t} \int_{\sigma}^{t}(t-s)^{-\gamma} x(s) d s=\frac{d}{d t}\left[I^{1-\gamma} x\right](t),
$$

is called the Riemann-Liouville fractional derivative [15, Definition 2.2], and the expression

$$
\left[{ }^{\mathrm{C}} D^{\gamma} x\right](t)=\frac{d}{d t}\left[I^{1-\gamma}(x-x(\sigma))\right](t),
$$

is called the Caputo fractional derivative [17, p. 91].

Definition 3 ([15, Definition 2.3]). We denote by $A^{\gamma}\left(T, R^{d}\right)$ the class of functions $x: T \rightarrow R^{d}$ representable by an integral of fractional order $\gamma \in(0,1)$ of a summable function: $x(t)=\left[I^{\gamma} \varphi\right](t), t \in T, \varphi \in L_{\infty}\left(T, R^{d}\right)$.

Remark 1. As follows from [15, Theorem 3.6] the operator $I^{\gamma}: L_{\infty}\left(T, R^{d}\right) \rightarrow C\left(T, R^{d}\right)$ is linear continuous. In addition, $\left[I^{\gamma} \varphi\right](\cdot) \in C\left(T, R^{d}\right)$ for an arbitrary $\varphi(\cdot) \in L_{\infty}\left(T, R^{d}\right)$.

The following classical inequalities will be used in the proofs (see, for example, [30, p. 2]):

- Cauchy's inequality with $\varepsilon$ :

$$
a b \leq \frac{\varepsilon}{2} a^{2}+\frac{1}{2 \varepsilon} b^{2},
$$

for each $a, b \in R$ and $\varepsilon>0$.

- Cauchy-Buniakovsky-Schwarz's inequality:

$$
\int_{M} f(t) g(t) d t \leq\|f\|_{L_{2}}\|g\|_{L_{2}}
$$

for each $f, g \in L_{2}(M)$. And in a finite-dimensional case:

$$
(x, y) \leq|x|_{n}|y|_{n},
$$

for each $x, y \in R^{n}$. 
Hereinafter, the symbol $|\cdot|_{n}:=\|\cdot\|_{R^{n}}$ stands for the Euclidean norm of a vector, and $(\cdot, \cdot)$ is the inner product in a finite-dimensional Euclidean space. For convenience, we do not mention the dimensions of Euclidean spaces in the inner product, this will not lead to a misunderstanding.

\section{Statement of the problem}

Consider a system of nonlinear differential equations

$$
\begin{aligned}
& {\left[{ }^{\mathrm{C}} D^{\gamma} x_{1}\right](t)=g_{1}\left(t, x_{1}\right)+B x_{2}(t),} \\
& {\left[{ }^{\mathrm{C}} D^{\gamma} x_{2}\right](t)=g_{2}\left(t, x_{1}, x_{2}\right)+C u(t),}
\end{aligned}
$$

with initial conditions

$$
x_{1}(\sigma)=x_{1 \sigma}, \quad x_{2}(\sigma)=x_{2 \sigma} .
$$

Here, $\gamma \in J:=(1 / 2,1), t \in T:=[\sigma, \theta]$ is a finite time interval, $x_{1} \in R^{n}, x_{2} \in R^{m}, x_{\sigma}=\left\{x_{1 \sigma}, x_{2 \sigma}\right\} \in R^{d}$ is the initial state, $d:=m+n, B$ and $C$ are constant matrices of corresponding dimensions $R^{n \times m}$ and $R^{m \times p}, u(\cdot)$ is a disturbance, $g_{1}: T \times R^{n} \rightarrow R^{n}$ and $g_{2}: T \times R^{n} \times R^{m} \rightarrow R^{m}$ are given vector functions satisfying the Lipschitz condition with constant $L>0$ with respect to variables $\left(t, x_{1}, x_{2}\right)$; i.e., the inequality

$$
\begin{aligned}
& \max \left\{\left|g_{1}\left(t_{1}, x_{1}\right)-g_{1}\left(t_{2}, y_{1}\right)\right|_{n} ;\left|g_{2}\left(t_{1}, x_{1}, x_{2}\right)-g_{2}\left(t_{2}, y_{1}, y_{2}\right)\right|_{m}\right\} \\
& \leq L\left(\left|t_{1}-t_{2}\right|+\left|x_{1}-y_{1}\right|_{n}+\left|x_{2}-y_{2}\right|_{m}\right),
\end{aligned}
$$

holds for any $\left(t_{1}, x_{1}, x_{2}\right),\left(t_{2}, y_{1}, y_{2}\right) \in T \times R^{n} \times R^{m}$. The external impact $u(\cdot)$ is a Lebesgue measurable function and is subject to an a priori restriction: $u(t) \in P$ for a. a. $t \in T$, where $P$ is a convex bounded and closed set in $R^{p}$.

The choice of systems of the form (4), (5) for the study is motivated by the fact that their particular cases are considered in modeling of complex economical and epidemiological processes. For instance, the complex energy supplydemand system [31] and the dynamics of tuberculosis virus [32], where some parameters of the systems may naturally be inaccessible for observation, as in the case we are considering.

Definition 4. A function $x: T \rightarrow R^{d}$ is called a solution to Cauchy problem (4)-(6), starting at a point $\left(\sigma, x_{\sigma}\right)$, if $x(\cdot) \in$ $A C^{\gamma}\left(T, R^{d}\right), x(\sigma)=x_{\sigma}$ and equalities (4), (5) hold for a. a. $t \in T$.

The problem in question is as follows. The trajectory of the system $x(t)=\left\{x_{1}(t), x_{2}(t)\right\}$ is unknown in advance and is determined by an impact $u(\cdot)$, which is also not specified, but the set $P$ is known.

At discrete times $\tau_{i} \in T, \tau_{i}<\tau_{i+1}$, the coordinate $x_{1}$ is measured with an error $h \in(0,1)$. The measurement results are vectors $\xi_{i}^{h} \in R^{n}$ satisfying the inequalities

$$
\left|x_{1}\left(\tau_{i}\right)-\xi_{i}^{h}\right|_{n} \leq h \text {. }
$$

It is required to find the disturbance $u(\cdot)$ generating $x(\cdot)$. The presence of an error in the measurements, the possible non uniqueness of the external impact, all these assumptions lead to the impossibility of exact finding the external impact acting on the object of study. In other words, the problem under consideration is reduced to constructing an algorithm for computing an approximation of $u(\cdot)$. The smaller is the measurement error $h$ and the finer is the mesh of the interval $T$ with the nodes $\tau_{i}$, the better should be this approximation.

\section{Solution algorithm}

Due to the incompleteness of the information (namely, the possibility of measuring at the time $\tau_{i}$ not whole phase state of the system $\left\{x_{1}\left(\tau_{i}\right), x_{2}\left(\tau_{i}\right)\right\}$, but only its part $\left.x_{1}\left(\tau_{i}\right)\right)$ the algorithm for solving the problem consists of two parallel working blocks of dynamical reconstruction. The first block is for reconstructing the unknown coordinate $x_{2}$. The information obtained as a result of its work passes to the second block, which in turn forms a certain approximation of $u(\cdot)$ according to the feedback law.

Before describing the algorithm for solving the problem, we give auxiliary constructions. We fix the uniform mesh $\Delta^{h}$ of the interval $T$ by the times $\tau_{i}^{h}$ :

$$
\Delta^{h}=\left\{\tau_{i}^{h}\right\}_{i=0}^{\kappa_{h}}, \quad \tau_{0}^{h}=\sigma, \quad \tau_{i+1}^{h}=\tau_{i}^{h}+\delta(h), \quad \tau_{\kappa_{h}}^{h}=\theta, \quad \delta(h)=\frac{\theta-\sigma}{\kappa_{h}} .
$$

Along with system (4), (5), we introduce an auxiliary system (a model)

$$
\begin{aligned}
& {\left[{ }^{\mathrm{C}} D^{\gamma} w_{1}\right](t)=g_{1}\left(t, \xi^{h}\right)+B \zeta^{h}(t),} \\
& {\left[{ }^{\mathrm{C}} D^{\gamma} w_{2}\right](t)=g_{2}\left(t, \xi^{h}, \zeta^{h}\right)+C v^{h}(t),}
\end{aligned}
$$

with the initial conditions

$$
w_{1}(\sigma)=x_{1 \sigma}, \quad w_{2}(\sigma)=x_{2 \sigma} .
$$


Here, the functions $\zeta^{h}(\cdot)$ and $v^{h}(\cdot)$ play the role of controls. We assume that the coordinates $w_{1}$ are also measured in the times $\tau_{i}^{h}$ with the error $h$, and the measurement results $\psi_{i}^{h} \in R^{n}$ satisfy the inequalities

$$
\left|w_{1}\left(\tau_{i}^{h}\right)-\psi_{i}^{h}\right|_{n} \leq h, \quad i=1, \ldots, \kappa_{h} .
$$

Definition 4 implies the continuity of the solution to the Cauchy problem (4)-(6), which implies its boundedness, i.e., there exists a constant $K_{0}$ such that $|x(t, u)|_{d} \leq K_{0}$ for all $u(t) \in P, t \in T$. We introduce the set $E$ as the closed ball with center at zero and radius $K_{0}$, i.e.

$$
E:=\left\{\zeta \in R^{m}:|\zeta|_{m} \leq K_{0}\right\} .
$$

Then, the first reconstruction block, following [14], is represented by system (9), in which the control action $\zeta^{h}$ is generated according to the rule

$$
\begin{aligned}
& \zeta^{h}(t)=\zeta_{i}^{h}, \quad t \in\left[\tau_{i}^{h}, \tau_{i+1}^{h}\right), \\
& \zeta_{i}^{h} \in \operatorname{argmin}\left\{\alpha_{1}(h)|\zeta|_{m}^{2}+\left(\psi_{i}^{h}-\xi_{i}^{h}, B \zeta\right): \zeta \in E\right\}, \quad i=0, \ldots, \kappa_{h}-1 .
\end{aligned}
$$

The second reconstruction block corresponds to the system (10) and the following rule of control formation

$$
\begin{aligned}
& v^{h}(t)=v_{i}^{h}, \quad t \in\left[\tau_{i}^{h}, \tau_{i+1}^{h}\right), \\
& v_{i}^{h} \in \operatorname{argmin}\left\{\alpha_{2}(h)|v|_{p}^{2}+\left(w_{2}\left(\tau_{i}\right)-\zeta_{i}^{h}, C v\right): v \in P\right\}, \quad i=0, \ldots, \kappa_{h}-1 .
\end{aligned}
$$

We proceed directly to the description of the algorithm for solving the problem under consideration.

Before the algorithm starts, in its preparatory part, we fix an error value $h$, numbers $\alpha_{1}=\alpha_{1}(h), \alpha_{2}=\alpha_{2}(h)$ and a mesh $\Delta^{h}$. The operation of the algorithm begins at the time $\sigma$, simultaneously with the system. The algorithm consists of the same type of steps, each of them is carried out on the interval $\left[\tau_{i}^{h}, \tau_{i+1}^{h}\right)$. The following actions are performed at the ith step:

(1) according to the observations $\xi_{i}^{h}$ and $\psi_{i}^{h}$, the control $\zeta_{i}^{h}$ is calculated by rule (12);

(2) the control $v_{i}^{h}$ is calculated by rule (13);

(3) under the action of this control $v_{i}^{h}$, system (9) passes from the state $w\left(\tau_{i}^{h}\right)$ to the state $w\left(\tau_{i+1}^{h}\right)=w\left(\tau_{i+1}^{h} ; \tau_{i}^{h}\right.$, $\left.w\left(\tau_{i}^{h}\right), v_{i}^{h}\right)$.

The algorithm ends at the time $\theta$.

To prove the main result of the paper, we need a number of auxiliary statements. We introduce the set

$$
U(x(\cdot)):=\left\{v \in L_{2}\left(T, R^{p}\right): v(t) \in P, \quad x\left(t ; \sigma, x_{\sigma}, v\right)=x(t), \quad t \in T\right\}
$$

of all admissible disturbances generating the same trajectory $x(t), t \in T$, of system $(4)$. It is easy to show that $U(x(\cdot))$ is convex bounded and closed due to the properties of the set $P$. Considering the fact that the trajectory $x(t)$ can be generated by the non-unique control $v$, we adhere to the concept of normal solution to inverse problems. The control $v=v_{*}$, following [1], is called normal if

$$
v_{*} \in U(x(\cdot)), \quad\left\|v_{*}\right\|_{L_{2}}:=\inf _{v \in U(x(\cdot))}\|v\|_{L_{2}} .
$$

The following lemma holds.

Lemma 1 ([14, Theorem 1]). For Cauchy problem (4)-(6), the following assertions are satisfied:

(i) For each bounded measurable control $u(t)\left(u \in L_{\infty}\left(T, R^{p}\right)\right)$ and each initial point $x_{\sigma} \in R^{d}$ there exists a unique solution $x=x(t, u)$ of problem (4)-(6) on the interval $T$.

(ii) There exist positive constants $K_{0}$ and $K_{1}>0$ such that

$$
\begin{aligned}
& |x(t, u)|_{d} \leq K_{0} \quad \forall u \in U, \quad \forall t \in T, \\
& \left|\left[{ }^{\mathrm{C}} D^{\gamma} x(\cdot, u)\right](t)\right|_{d} \leq K_{1} \quad \forall u \in U, \quad \text { for a.a. } \quad t \in T .
\end{aligned}
$$

(iii) If $\gamma \in J$, then there exists a constant $M_{0}>0 \forall t_{1}, t_{2} \in T, \forall u_{1}, u_{2} \in U$ such that

$$
\left|x\left(t_{1}, u_{1}\right)-x\left(t_{2}, u_{2}\right)\right|_{d} \leq M_{0}\left(\left|t_{2}-t_{1}\right|^{\gamma}+\left\|u_{1}-u_{2}\right\|_{L_{2}}\right) .
$$

(iv) If a sequence $\left\{u_{k}\right\} \in U$ converges to a control $u(\cdot)$ weakly in $L_{2}\left(T, R^{p}\right)$, then

$$
\left\|x\left(t, u_{k}\right)-x(t, u)\right\|_{c} \rightarrow 0,
$$

i.e., the trajectories $\left\{x\left(t, u_{k}\right)\right\}$ converge to $x(t, u)$ uniformly on the interval T. Here, $\|x\|_{C}:=\max _{t \in T}|x(t)|_{d}$ is the norm in the space of continuous functions $C\left(T, R^{d}\right)$. 
The action of the first reconstruction block is represented by the following two lemmas.

Lemma 2 ([14, Theorem 2]). Let there exist functions $\alpha_{1}(h)$ and $\delta(h)$ such that $\alpha_{1}(h) \rightarrow 0$ and $\delta(h) \rightarrow 0$ as $h \rightarrow 0$. Then, one can specify closed-form expressions for constants $d_{i}>0$, independent of $h, \alpha_{1}$ and $\delta$, such that the inequality

$$
\left|w_{1}(t)-x_{1}(t)\right|_{n}^{2} \leq d_{1} h+d_{2} \alpha_{1}+d_{3} \delta^{\gamma}, \quad t \in T,
$$

holds for solutions of systems (4) and (9).

Condition 1. There exist functions $\alpha_{1}(h), \delta(h)$ and a number $\beta_{1} \in(0,1)$ such that

$$
\alpha_{1}(h) \rightarrow 0, \quad \delta(h) \rightarrow 0, \quad \frac{\alpha_{1}(h)+\delta^{\gamma}(h)}{h^{\beta_{1}}} \rightarrow 0, \quad \frac{h+\delta^{\gamma}(h)}{\alpha_{1}(h)} \rightarrow 0 \quad \text { as } \quad h \rightarrow 0 .
$$

Lemma 3 ([14, Theorem 4]). Let Condition 1 be satisfied, and $m=n$, det $B \neq 0$. Then, the estimate

$$
\left\|x_{2}-\zeta^{h}\right\|_{L_{2}}^{2} \leq \frac{d_{1} h+d_{2} \alpha_{1}+d_{3} \delta^{\gamma}}{h^{\beta_{1}}}+\frac{d_{4} h+d_{5} \delta^{\gamma}}{\alpha_{1}}
$$

holds. Here the constants $d_{i}>0, i=1, \ldots, 5$, are independent of $h, \alpha_{1}$ and $\delta$.

Passing to the second reconstruction block, Condition 1 representing the concordance of the mesh step $\delta$, the regularization parameter $\alpha_{1}$ and the measurement error $h$, needs to be strengthened.

Condition 2. There exist functions $\alpha_{1}(h), \alpha_{2}(h), \delta(h)$ and numbers $\beta_{1}, \beta_{2} \in(0,1)$ such that

$$
\begin{aligned}
& \alpha_{1}(h) \rightarrow 0, \quad \alpha_{2}(h)=h^{\beta_{2}} \rightarrow 0, \quad \delta(h) \rightarrow 0, \\
& \frac{h+\alpha_{1}(h)+\delta^{\gamma}(h)}{\alpha_{2} h^{\beta_{1}}} \rightarrow 0, \quad \frac{h+\delta^{\gamma}}{\alpha_{1} \alpha_{2}} \rightarrow 0 \text { for } h \rightarrow 0 .
\end{aligned}
$$

Remark 2. The set of parameters $\beta_{1}=\frac{1}{10}, \beta_{2}=\frac{3}{10}, \alpha_{1}(h)=h^{3 / 5}, \alpha_{2}(h)=h^{3 / 10}, \delta(h)=h^{1 / \gamma}$ satisfies Condition 2 .

Lemma 4. Suppose that Condition 2 is fulfilled and $m=n$, det $B \neq 0$. Then, for systems (5) and (10), the inequalities

$$
\begin{aligned}
& \left\|v^{h}\right\|_{L_{2}}^{2} \leq\|u\|_{L_{2}}^{2}+\frac{\bar{d}_{1} h+\bar{d}_{2} \delta^{\gamma}}{\alpha_{2}}+\frac{\bar{d}_{3} h+\bar{d}_{4} \alpha_{1}+\bar{d}_{5} \delta^{\gamma}}{\alpha_{2} h^{\beta_{1}}}+\frac{\bar{d}_{6} h+\bar{d}_{7} \delta^{\gamma}}{\alpha_{1} \alpha_{2}}, \\
& \left|w_{2}(t)-x_{2}(t)\right|_{m}^{2} \leq d_{1} \alpha_{2}+d_{2} \delta^{\gamma}+\frac{d_{3} h+d_{4} \alpha_{1}+d_{5} \delta^{\gamma}}{\alpha_{2} h^{\beta_{1}}}+\frac{d_{6} h+d_{7} \delta^{\gamma}}{\alpha_{1} \alpha_{2}}
\end{aligned}
$$

hold, where the constants $\bar{d}_{i}>0$ and $d_{i}>0, i=1, \ldots, 7$, are independent of $h, \alpha_{1}, \alpha_{2}$ and $\delta$.

Proof. At first, we prove the inequality (17). Let $t \in\left[\tau_{i}^{h}, \tau_{i+1}^{h}\right), i=0, \ldots, \kappa_{h}-1$. Writing $\mu_{2}(t):=w_{2}(t)-x_{2}(t)$ and considering the difference between systems (10) and (5), we obtain

$$
\left[{ }^{\mathrm{C}} D^{\gamma} \mu_{2}\right](t)=g_{2}\left(t, \xi^{h}, \zeta^{h}\right)-g_{2}\left(t, x_{1}, x_{2}\right)+C\left(v^{h}(t)-u(t)\right) .
$$

Let us use the estimate in [33, Lemma 4.1], according to (11), i.e., $\mu_{2}(\sigma)=0$, and rewrite it in more convenient terms as

$$
\frac{1}{2}\left[{ }^{\mathrm{C}} D^{\gamma}\left|\mu_{2}\right|_{m}^{2}\right](t) \leq\left(\left[{ }^{\mathrm{C}} D^{\gamma} \mu_{2}\right](t), \mu_{2}(t)\right) .
$$

The latter inequality, in virtue of (19), can be represented as

$$
\begin{aligned}
\frac{1}{2}\left[{ }^{\mathrm{C}} D^{\gamma}\left|\mu_{2}\right|_{m}^{2}\right](t) \leq & \left(g_{2}\left(t, \xi^{h}, \zeta^{h}\right)-g_{2}\left(t, x_{1}, x_{2}\right), \mu_{2}(t)\right) \\
& +\left(C\left(v^{h}(t)-u(t)\right), \mu_{2}(t)\right) .
\end{aligned}
$$

Let us estimate the first term on the right-hand side of (20). Using Cauchy-Buniakovsky-Schwarz's inequality (3), the Lipschitz condition (7), as well as (8), (14) and (15), we get

$$
\begin{aligned}
\left(g_{2}\left(t, \xi^{h}, \zeta^{h}\right)-g_{2}\left(t, x_{1}, x_{2}\right), \mu_{2}(t)\right) & \leq\left|g_{2}\left(t, \xi^{h}, \zeta^{h}\right)-g_{2}\left(t, x_{1}, x_{2}\right)\right|_{m}\left|\mu_{2}(t)\right|_{m} \\
& \leq L\left(\left|\xi^{h}(t)-x_{1}\left(\tau_{i}^{h}\right)\right|_{n}+\left|x_{1}\left(\tau_{i}^{h}\right)-x_{1}(t)\right|_{n}+\left|\zeta^{h}(t)-x_{2}(t)\right|_{m}\right)\left(\left|w_{2}(t)\right|_{m}+\left|x_{2}(t)\right|_{m}\right) \\
& \leq 2 K_{0} L\left(h+M_{0} \delta^{\gamma}+\left|\zeta^{h}(t)-x_{2}(t)\right|_{m}\right) .
\end{aligned}
$$


For the second term on the right-hand side of (20), given $v^{h}(t), u(t) \in P$ and applying Cauchy-Buniakovsky-Schwarz's inequality (3), the triangle inequality, and the estimate (15), we find

$$
\begin{aligned}
\left(C v(t), \mu_{2}(t)\right) & =\left(C v(t), w_{2}(t)-w_{2}\left(\tau_{i}^{h}\right)+w_{2}\left(\tau_{i}^{h}\right)-\zeta^{h}(t)+\zeta^{h}(t)-x_{2}(t)\right) \\
& \leq\left(C v(t), w_{2}(t)-w_{2}\left(\tau_{i}^{h}\right)\right)+\left(C \nu(t), w_{2}\left(\tau_{i}^{h}\right)-\zeta^{h}(t)\right)+\left(C v(t), \zeta^{h}(t)-x_{2}(t)\right) \\
& \leq c_{1} \delta^{\gamma}+\left(C v(t), w_{2}\left(\tau_{i}\right)-\zeta_{i}^{h}\right)+c_{2}\left|\zeta^{h}(t)-x_{2}(t)\right|_{m},
\end{aligned}
$$

where $v(t):=v^{h}(t)-u(t)$. Substituting expressions (21) and (22) into (20) and adding terms with the factor $\alpha_{2}$, we have

$$
\begin{aligned}
& \alpha_{2}\left|v^{h}(t)\right|_{p}^{2}-\alpha_{2}|u(t)|_{p}^{2}+\frac{1}{2}\left[{ }^{\mathrm{C}} D^{\gamma}\left|\mu_{2}\right|_{m}^{2}\right](t) \\
& \leq c_{3} h+c_{4} \delta^{\gamma}+c_{5}\left|\zeta^{h}(t)-x_{2}(t)\right|_{m}+\alpha_{2}\left|v^{h}(t)\right|_{p}^{2}-\alpha_{2}|u(t)|_{p}^{2} \\
& \quad+\left(C\left(v^{h}(t)-u(t)\right), w_{2}\left(\tau_{i}\right)-\zeta_{i}^{h}\right) .
\end{aligned}
$$

Rule (13) of the choice of control $v^{h}$ implies the relation

$$
\left(C\left(v^{h}(t)-u(t)\right), w_{2}\left(\tau_{i}\right)-\zeta_{i}^{h}\right)+\alpha_{2}\left|v^{h}(t)\right|_{p}^{2}-\alpha_{2}|u(t)|_{p}^{2} \leq 0 .
$$

Then, taking into account the latter inequality and the fact that $v^{h}(t), u(t) \in P$, we rewrite formula (23) as

$$
\begin{aligned}
& \alpha_{2}\left|v^{h}(t)\right|_{p}^{2}-\alpha_{2}|u(t)|_{p}^{2}+\frac{1}{2}\left[{ }^{\mathrm{C}} D^{\gamma}\left|\mu_{2}\right|_{m}^{2}\right](t) \\
& \leq c_{3} h+c_{4} \delta^{\gamma}+c_{5}\left|\zeta^{h}(t)-x_{2}(t)\right|_{m} .
\end{aligned}
$$

By calculating the integral of the right- and left-hand sides of formula (24):

$$
\begin{aligned}
& \alpha_{2}\left[I^{1}\left|v^{h}\right|_{p}^{2}\right](t)-\alpha_{2}\left[I^{1}|u|_{p}^{2}\right](t)+\frac{1}{2}\left[I^{1}\left({ }^{\mathrm{C}} D^{\gamma}\left|\mu_{2}\right|_{m}^{2}\right)\right](t) \\
& \leq c_{6} h+c_{7} \delta^{\gamma}+c_{5}\left[I^{1}\left|\zeta^{h}-x_{2}\right|_{m}\right](t),
\end{aligned}
$$

where $\left[I^{1} x\right](t):=\int_{0}^{t} x(s) d s$. Based on [17, Lemma 2.3], as well as on Definitions 3, 4 and [17, Lemma 2.5], we get

$$
\left[I^{1}\left({ }^{\mathrm{C}} D^{\gamma}\left|\mu_{2}\right|_{m}^{2}\right)\right](t)=\left[I^{1-\gamma} I^{\gamma}\left({ }^{\mathrm{C}} D^{\gamma}\left|\mu_{2}\right|_{m}^{2}\right)\right](t)=\left[I^{1-\gamma}\left|\mu_{2}\right|_{m}^{2}\right](t),
$$

and since $\left|\mu_{2}(t)\right|_{m}^{2} \geq 0, \forall t \in T$, we deduce that

$$
\left[I^{1-\gamma}\left|\mu_{2}\right|_{m}^{2}\right](t) \geq 0, \quad \forall t \in T .
$$

Using (26) in (25), we have the expression

$$
\alpha_{2}\left\|v^{h}\right\|_{L_{2}}^{2}-\alpha_{2}\|u\|_{L_{2}}^{2} \leq c_{6} h+c_{7} \delta^{\gamma}+c_{5}\left\|\zeta^{h}-x_{2}\right\|_{L_{2}}^{2},
$$

whence, taking into account the result of Lemma 3, formula (16), we obtain estimate (17).

Now we turn to the proof of inequality (18). Since $v^{h}(t), u(t) \in P$, expression (24) can be written as

$$
\left[{ }^{\mathrm{C}} D^{\gamma}\left|\mu_{2}\right|_{m}^{2}\right](t) \leq 2\left[{ }^{\mathrm{C}} D^{\gamma} I^{\gamma}\left(c_{3} h+c_{6} \alpha_{2}+c_{4} \delta^{\gamma}\right)\right](t)+2 c_{5}\left[{ }^{\mathrm{C}} D^{\gamma} I^{\gamma}\left|\zeta^{h}-x_{2}\right|_{m}\right](t) .
$$

Further, using the Fractional Comparison Principle [34, Lemma 6.1], we derive

$$
\left|\mu_{2}(t)\right|_{m}^{2} \leq c_{7} h+c_{8} \alpha_{2}+c_{9} \delta^{\gamma}+2 c_{5}\left[I^{\gamma}\left|\zeta^{h}-x_{2}\right|_{m}\right](t) .
$$

For the last term on the right-hand side of (27) we use Cauchy-Buniakovsky-Schwarz's inequality (2). Then, in view of Cauchy's inequality (1) with $\varepsilon=h^{\beta_{2}}$ and $\gamma \in J$, we get

$$
\begin{aligned}
{\left[I^{\gamma}\left|\zeta^{h}-x_{2}\right|_{m}\right](t) } & =\frac{1}{\Gamma(\gamma)} \int_{\sigma}^{t}(t-s)^{\gamma-1}\left|\zeta^{h}(s)-x_{2}(s)\right|_{m} d s \\
& \leq \frac{1}{\Gamma(\gamma)}\left(\int_{\sigma}^{t}(t-s)^{2 \gamma-2} d s\right)^{1 / 2}\left\|\zeta^{h}-x_{2}\right\|_{L_{2}} \\
& \leq \frac{(t-\sigma)^{\gamma-1 / 2}}{(2 \gamma-1)^{1 / 2} \Gamma(\gamma)}\left\|\zeta^{h}-x_{2}\right\|_{L_{2}} \\
& \leq c_{10} h^{\beta_{2}}+h^{-\beta_{2}}\left\|\zeta^{h}-x_{2}\right\|_{L_{2}}^{2} .
\end{aligned}
$$

Substituting estimate (16) in (28), we find

$$
\left[I^{\gamma}\left|\zeta^{h}-x_{2}\right|_{m}\right](t) \leq c_{10} h^{\beta_{2}}+\frac{c_{11} h+c_{12} \alpha_{1}+c_{13} \delta^{\gamma}}{h^{\beta_{1}+\beta_{2}}}+\frac{c_{14} h+c_{15} \delta^{\gamma}}{\alpha_{1} h^{\beta_{2}}} .
$$

Using inequality (29) in (27), we obtain formula (18). Lemma is proved. 
One can establish the following result based on Lemmas 1, 2, and 4 similar to the proof of Theorem 3 from [14].

Theorem 1. Suppose Condition 2 is satisfied and $m=n$, det $B \neq 0$. Then, for the control action $v^{h}$ given by rule (13), one has the convergence $v^{h} \rightarrow v_{*}$ in $L_{2}\left(T, R^{p}\right)$ as $h \rightarrow 0$, where $v_{*}$ is a normal control.

\section{Convergence rate of the algorithm}

One can estimate the convergence rate on the finite time interval $T$. Toward this aim, we need the following assertion.

Lemma 5 ([6, p. 47]). If a bounded function $\psi: T \rightarrow R^{p}$ and a function $\eta: T \rightarrow R^{p}$ of bounded variation satisfy the inequalities

$$
\left|\int_{\sigma}^{t} \psi(\tau) d \tau\right|_{p} \leq \varepsilon, \quad|\eta(t)|_{p} \leq p
$$

for any $t \in T$, then

$$
\left|\int_{\sigma}^{t}(\eta(\tau), \psi(\tau)) d \tau\right| \leq \varepsilon\left(\operatorname{var}_{T} \eta+p\right)
$$

where $\operatorname{var}_{T} \eta$ is the total variation of $\eta$ on the interval $T$.

Theorem 2. Let Condition 2 be satisfied, $m=n=p$, $\operatorname{det} B \neq 0$, $\operatorname{det} C \neq 0$ and let there exist numbers $\beta_{3}, \beta_{4} \in(0,1)$ such that $\rho_{1}(h) \rightarrow 0, \rho_{2}(h) \rightarrow 0$ as $h \rightarrow 0$. Then, one can specify closed-form expressions for constants $\tilde{d}_{i}(i=1, \ldots, 8), \hat{d}_{j}$ $(j=1, \ldots, 6), \bar{d}_{k}(k=1, \ldots, 7)$, independent of $h, \alpha_{1}, \alpha_{2}$ and $\delta$ such that the inequality

$$
\left\|u-v^{h}\right\|_{L_{2}}^{2} \leq \rho_{1}(h)+\rho_{2}(h)+\rho_{3}(h)
$$

holds. Here,

$$
\begin{aligned}
& \rho_{1}(h):=\tilde{d}_{1} h^{\beta_{3}}+\frac{\tilde{d}_{2} \alpha_{2}+\tilde{d}_{3} \delta^{\gamma}}{h^{\beta_{3}}}+\frac{\tilde{d}_{4} h+\tilde{d}_{5} \alpha_{1}+\tilde{d}_{6} \delta^{\gamma}}{\alpha_{2} h^{\beta_{1}+\beta_{3}}}+\frac{\tilde{d}_{7} h+\tilde{d}_{8} \delta^{\gamma}}{\alpha_{1} h^{\beta_{1}+\beta_{3}}}, \\
& \rho_{2}(h):=\hat{d}_{1} h^{\beta_{4}}+\frac{\hat{d}_{2} h+\hat{d}_{3} \alpha_{1}+\hat{d}_{4} \delta^{\gamma}}{h^{\beta_{1}+\beta_{4}}}+\frac{\hat{d}_{5} h+\hat{d}_{6} \delta^{\gamma}}{\alpha_{1} h^{\beta_{4}}}, \\
& \rho_{3}(h):=\frac{\bar{d}_{1} h+\bar{d}_{2} \delta^{\gamma}}{\alpha_{2}}+\frac{\bar{d}_{3} h+\bar{d}_{4} \alpha_{1}+\bar{d}_{5} \delta^{\gamma}}{\alpha_{2} h^{\beta_{1}}}+\frac{\bar{d}_{6} h+\bar{d}_{7} \delta^{\gamma}}{\alpha_{1} \alpha_{2}} .
\end{aligned}
$$

Proof. Let $t \in\left[\tau_{i}^{h}, \tau_{i+1}^{h}\right), i=0, \ldots, \kappa_{h}-1$. Using formulas (5) and (10), we estimate the integral

$$
\begin{aligned}
& \left|\int_{\sigma}^{t} C\left(v^{h}(\tau)-u(\tau)\right) d \tau\right|_{m} \\
& =\left|\int_{\sigma}^{t}\left(\left[{ }^{\mathrm{C}} D^{\gamma} w_{2}\right](\tau)-g_{2}\left(\tau, \xi^{h}, \zeta^{h}\right)-\left[{ }^{\mathrm{C}} D^{\gamma} x_{2}\right](\tau)+g_{2}\left(\tau, x_{1}, x_{2}\right)\right) d \tau\right|_{m} \\
& \leq I_{1}+I_{2}
\end{aligned}
$$

where

$$
\begin{aligned}
& I_{1}:=\left|\int_{\sigma}^{t}\left[{ }^{c} D^{\gamma}\left(w_{2}-x_{2}\right)\right](\tau) d \tau\right|_{m}, \\
& I_{2}:=\left|\int_{\sigma}^{t}\left(g_{2}\left(\tau, x_{1}, x_{2}\right)-g_{2}\left(\tau, \xi^{h}, \zeta^{h}\right)\right) d \tau\right|_{m} .
\end{aligned}
$$

Transforming expression (32) using [17, Lemma 2.3], [34, Lemma 3.1], as well as Cauchy's inequality (1) with $\varepsilon=h^{\beta_{3}}$, we have

$$
\begin{aligned}
I_{1} & =\left|\left[I^{1-\gamma}\left(w_{2}-x_{2}\right)\right](t)\right|_{m} \leq\left[I^{1-\gamma}\left|w_{2}-x_{2}\right|_{m}\right](t) \\
& \leq \frac{1}{2}\left[I^{1-\gamma}\left(h^{\beta_{3}}+h^{-\beta_{3}}\left|w_{2}-x_{2}\right|_{m}^{2}\right)\right](t) .
\end{aligned}
$$

Taking into account estimate (18) in the latter formula, we obtain

$$
I_{1} \leq \rho_{1}(h)
$$


We estimate expression (33), using Lipschitz condition (7), formulas (8), (15) and Cauchy's inequality (1) with $\varepsilon=h^{\beta_{4}}$, we find

$$
\begin{aligned}
I_{2} & \leq \int_{\sigma}^{t}\left|g_{2}\left(\tau, x_{1}, x_{2}\right)-g_{2}\left(\tau, \xi^{h}, \zeta^{h}\right)\right|_{m} d \tau \\
& \leq L \int_{\sigma}^{t}\left(\left|x_{1}(\tau)-x_{1}\left(\tau_{i}^{h}\right)\right|_{n}+\left|x_{1}\left(\tau_{i}^{h}\right)-\xi^{h}(\tau)\right|_{n}+\left|x_{2}(\tau)-\zeta^{h}(\tau)\right|_{m}\right) d \tau \\
& \leq L(\theta-\sigma) h+\frac{1}{2} L(\theta-\sigma) h^{\beta_{4}}+\frac{1}{2} L h^{-\beta_{4}}\left\|\zeta^{h}-x_{2}\right\|_{L_{2}}^{2} .
\end{aligned}
$$

Recalling the formula (16) in the latter expression, we get the inequality

$$
I_{2} \leq \rho_{2}(h) .
$$

Using (34) and (35) in (31), we derive

$$
\left|\int_{\sigma}^{t} C\left(v^{h}(\tau)-u(\tau)\right) d \tau\right|_{m} \leq \rho_{1}(h)+\rho_{2}(h) .
$$

Let $\langle\cdot, \cdot\rangle$ denote the inner product in $L_{2}\left(T, R^{p}\right)$, then, taking into account (17), we find

$$
\begin{aligned}
\left\|u-v^{h}\right\|_{L_{2}}^{2} & =\|u\|_{L_{2}}^{2}-2\left\langle u, v^{h}\right\rangle+\left\|v^{h}\right\|_{L_{2}}^{2} \\
& \leq 2\|u\|_{L_{2}}^{2}-2\left\langle u, v^{h}\right\rangle+\rho_{3}(h) \\
& =2\left\langle u-v^{h}, u\right\rangle+\rho_{3}(h) .
\end{aligned}
$$

Following Lemma 5, we get

$$
\left\|u-v^{h}\right\|_{L_{2}}^{2} \leq \rho_{1}(h)+\rho_{2}(h)+\rho_{3}(h) .
$$

This completes the proof of theorem.

Remark 3. For the set of parameters specified in Remark 2 and $\beta_{3}=\beta_{4}=\frac{1}{5}$, estimate (30) takes the form

$$
\left\|u-v^{h}\right\|_{L_{2}}^{2} \leq C_{1} h^{1 / 5},
$$

where the constant $C_{1}>0$ is independent of $h, \alpha_{1}, \alpha_{2}$ and $\delta$.

\section{Numerical example}

We test the proposed algorithm for reconstructing an external impact on a specific dynamical system in real-time mode. The system of fractional differential equations

$$
\begin{aligned}
& {\left[{ }^{\mathrm{C}} D^{\gamma} x\right](t)=0.5 x(t)+g(t)-y(t), \quad \gamma=0.6,} \\
& {\left[{ }^{\mathrm{C}} D^{\gamma} y\right](t)=x(t)-t y(t)-0.5+u(t), \quad t \in T=[0,0.5],}
\end{aligned}
$$

with the initial conditions

$$
x(0)=0.5, \quad y(0)=0,
$$

where

$$
\begin{aligned}
g(t)= & t^{2 \gamma}-0.5 t^{1+2 \gamma}-t^{2}+0.5 t-0.25 \\
& -\frac{t^{\gamma}(1+\gamma) \Gamma(1+2 \gamma)}{2 \Gamma(2+\gamma)}+\frac{t^{1-\gamma}(4 t-2+\gamma)}{(2-\gamma)(1-\gamma) \Gamma(1-\gamma)} \\
& +\frac{4^{2 \gamma} t^{1+\gamma} \Gamma(1.5+\gamma)+\sqrt{\pi}(1+\gamma) \Gamma(1+2 \gamma)}{\sqrt{\pi}(1+\gamma) \Gamma(1+2 \gamma)},
\end{aligned}
$$

is considered. If the disturbance is given by

$$
u(t)=t-2 t^{2}, \quad t \in T,
$$

belonging to the set $P=[-1,1]$, then the exact solution to Cauchy problem (36)-(38) is determined by the formulas

$$
\begin{aligned}
& x(t)=\frac{t^{\gamma}(1+\gamma) \Gamma(1+2 \gamma)}{\Gamma(2+\gamma)}+t^{2 \gamma+1}+2 t^{2}-t+0.5, \\
& y(t)=t^{2 \gamma} .
\end{aligned}
$$




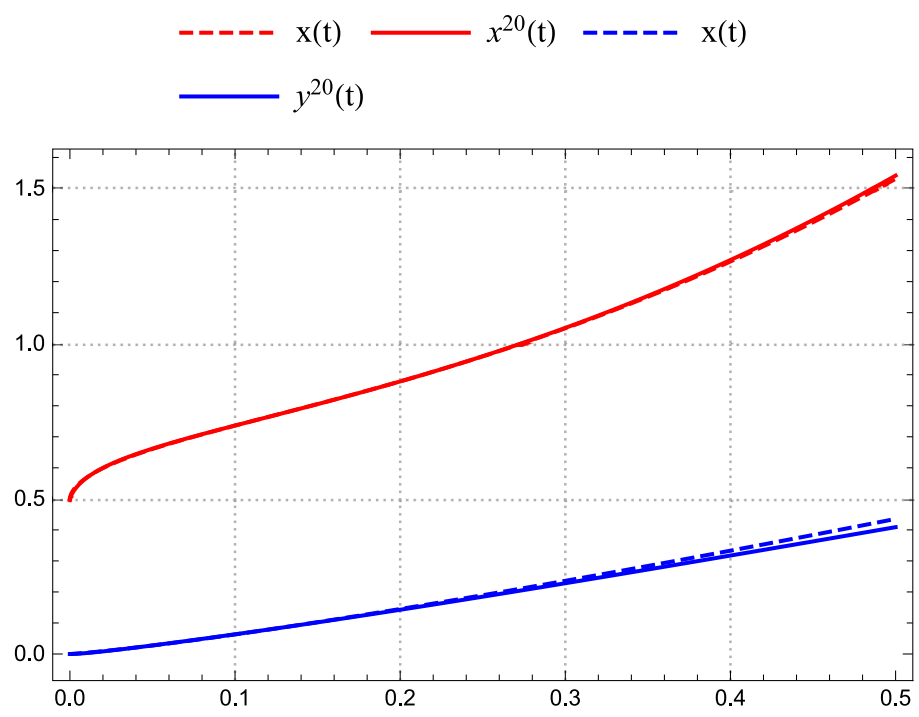

Fig. 1. Components $x(t), y(t)$ of the exact solution and $x^{20}(t), y^{20}(t)$ of the approximate solution to Cauchy problem (36)-(38).

In this case, we have the following parameters of the system (4), (5):

$$
g_{1}(t, x)=x(t)-g(t), \quad g_{2}(t, x, y)=x(t)-t y(t)-0.5, \quad B=-1, \quad C=1 .
$$

We use the Variational iteration method (VIM) described in [35] to find an approximate solution to problem (36)-(38). The approximate solution plays the role of the exact solution measured with an error. Following this approach, the iterative formulas for system (36), (37) have the form

$$
\begin{gathered}
x^{k+1}(t)=x^{k}(t)-\int_{0}^{t}\left(\left[{ }^{\mathrm{C}} D^{\gamma} x^{k}\right](s)-\left(0.5 x^{k}(s)-y^{k}(s)+g(s)\right)\right) d s, \\
y^{k+1}(t)=y^{k}(t)-\int_{0}^{t}\left(\left[{ }^{\mathrm{C}} D^{\gamma} y^{k}\right](s)-\left(x^{k}(s)-s y^{k}(s)-0.5+u(s)\right)\right) d s, \\
x^{0}(t)=0.5, \quad y^{0}(t)=0 .
\end{gathered}
$$

Denote $\chi^{k}(t):=\left\{x^{k}(t), y^{k}(t)\right\}$. The criterion for stopping the iterative procedure consists in the fulfillment of the inequality

$$
\begin{aligned}
\left\|\chi^{k+1}-\chi^{k}\right\|_{C} & =\max _{t \in T}\left|\chi^{k+1}(t)-\chi^{k}(t)\right| \\
& =\max _{t \in T} \sqrt{\left(x^{k+1}(t)-x^{k}(t)\right)^{2}-\left(y^{k+1}(t)-y^{k}(t)\right)^{2}} \leq h .
\end{aligned}
$$

For example, for $h=0.1,5$ iterations are needed to achieve a given accuracy; for $h=0.001$, we need 20 iterations. Fig. 1 shows the exact solution to Cauchy problem (36)-(38) and the approximate solution at the 20th iteration.

Next, we define the reconstruction blocks. The equations of the model (9), (10) take the form

$$
\begin{aligned}
& {\left[{ }^{C} D^{\gamma} w_{1}\right](t)=0.5 \xi^{h}(t)+g(t)-\zeta^{h}(t),} \\
& {\left[{ }^{C} D^{\gamma} w_{2}\right](t)=\xi^{h}(t)-t \zeta^{h}(t)-0.5+v^{h}(t),}
\end{aligned}
$$

with initial conditions

$$
w_{1}(0)=0.5, \quad w_{2}(0)=0 .
$$

For controls (12) and (13), the smoothing functionals of the Tikhonov regularization method [14] are used, i.e., at the time moment $\tau_{i}^{h}=i \delta(h), i=1, \ldots, \kappa_{h}-1$, the values $\zeta_{i}^{h}$ and $v_{i}^{h}$ are determined by the formulas

$$
\begin{aligned}
& \zeta_{i}^{h} \in \operatorname{argmin}\left\{\alpha_{1}|\zeta|^{2}+\left(\xi_{i}^{h}-\psi_{i}^{h}, \zeta\right), \zeta \in E\right\}, \\
& v_{i}^{h} \in \operatorname{argmin}\left\{\alpha_{2}|v|^{2}+\left(w_{2}\left(\tau_{i}^{h}\right)-\zeta_{i}^{h}, v\right), v \in P\right\},
\end{aligned}
$$


- $w_{1}(\mathrm{t}) \cdot \xi^{h}(\mathrm{t})$

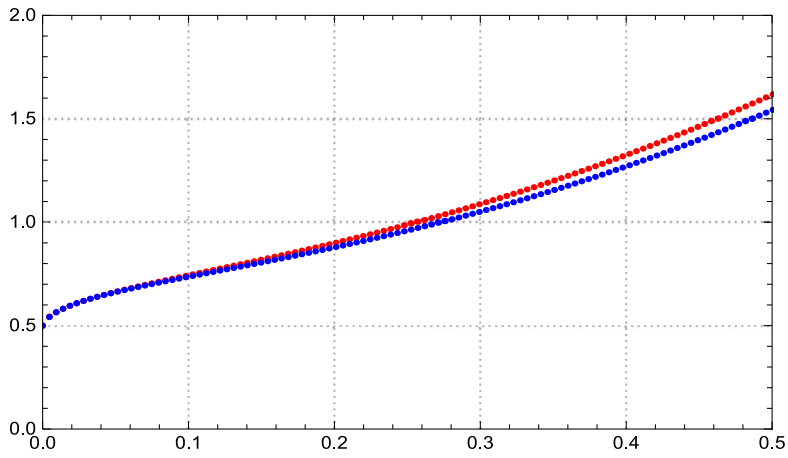

a)

- $v^{h}(\mathrm{t}) \cdot \mathrm{u}(\mathrm{t})$

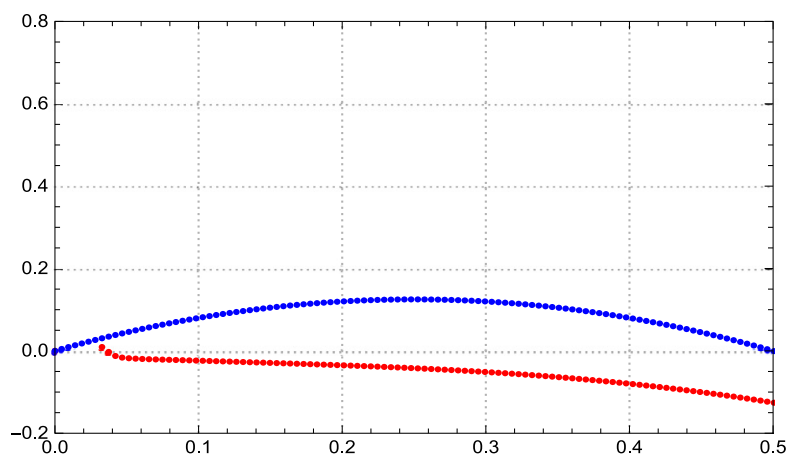

c)
- $w_{2}(\mathrm{t}) \cdot \zeta^{h}(\mathrm{t}) \cdot \mathrm{y}(\mathrm{t})$

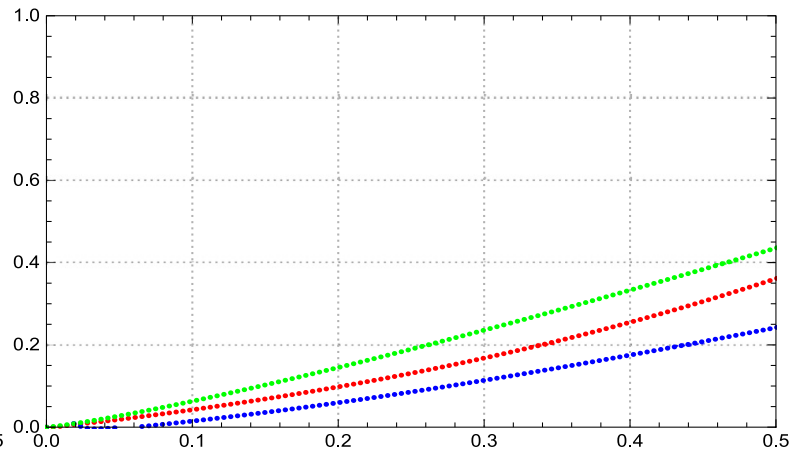

b) component $y(t)$ of system (37) and control $\zeta^{h}(t)$ of system (40); (c) control $u$ of system (37) and reconstructed control $v$ of system (41).

where $E=[-3,3]$. We define the vector $\xi^{h}$ as a result of applying iteration procedure (39) by the formulas

$$
\xi_{i}^{h}=x^{k}\left(\tau_{i}^{h}\right), \quad i=1, \ldots, \kappa_{h}-1 .
$$

We select the set of parameters as follows:

$$
\alpha_{1}(h)=h^{3 / 5}, \quad \alpha_{2}(h)=h^{3 / 10}, \quad \beta_{1}=\frac{1}{10}, \quad \beta_{2}=\frac{3}{10}, \quad \beta_{3}=\beta_{4}=\frac{1}{5}, \quad \delta(h)=h^{1 / \gamma} .
$$

The simulation results are presented in Fig. 2 for $h=0.04$ and in Fig. 3 for $h=0.02$.

\section{Conclusions}

The real-time external impact reconstruction problem for a system of nonlinear fractional differential equations with the Caputo derivative is considered. The problem is complicated by the lack of information about the system, namely, only a part of its coordinates are available for measuring with an error. We propose the solution algorithm resistant to informational noises and computational errors. Structurally, it consists of two reconstruction blocks interacting in real time, the missing information about the unobservable coordinate is obtained in the first block and supplied to the second one, which restores the unknown input. The action of each block is based on the combination of Krasovskii extremal shift method with regularization methods of the theory of ill-posed problems. The proposed methods of forming the control according to the feedback principle in each of the blocks make it possible to obtain a stable approximation of the disturbance, this is established in Theorem 1. It should be noted that the estimates obtained in Theorem 2, as well as in Remark 3, can be improved. The numerical example is considered that demonstrates the operation of the constructed algorithm for the specific system of fractional differential equations. Figs. 2, 3 show that, with a decrease in error rate, the external impact is reconstructed more accurately. A further development of the approach used to solve this problem is seen both in complication the form of the system under consideration, introducing fractional derivatives of different 
- $w_{1}(\mathrm{t}) \cdot \xi^{h}(\mathrm{t})$

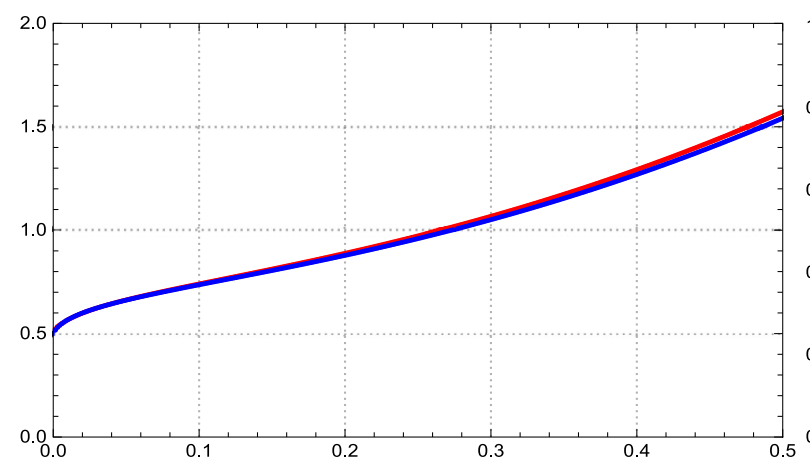

a)

$\cdot v^{h}(\mathrm{t}) \cdot \mathrm{u}(\mathrm{t})$

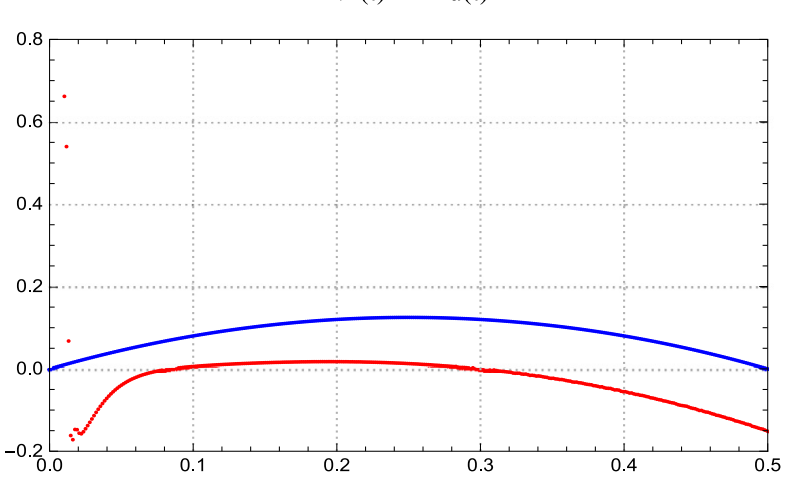

c) $\cdot w_{2}(\mathrm{t}) \cdot \zeta^{h}(\mathrm{t}) \cdot \mathrm{y}(\mathrm{t})$

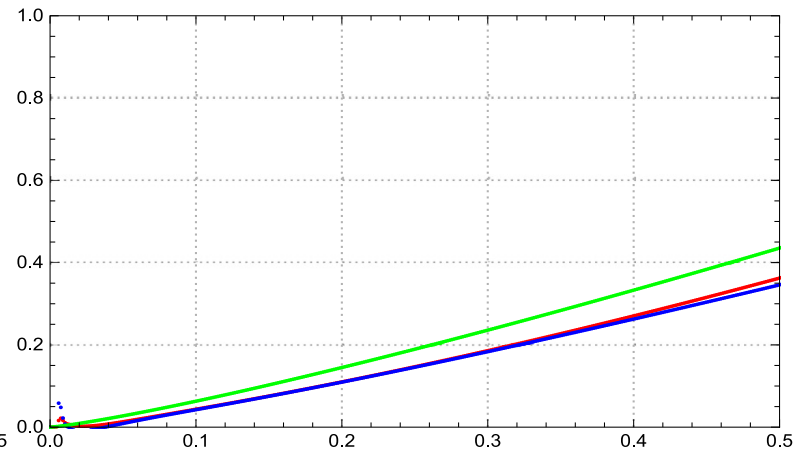

b)

Fig. 3. The simulation results for $h=0.02$ : (a) component $w_{1}(t)$ of system (40) and measurements $\xi^{h}(t)$ of $x(t)$; (b) component $w_{2}(t)$ of system (41), component $y(t)$ of system (37) and control $\zeta^{h}(t)$ of system (40); (c) control $u$ of system (37) and reconstructed control $v$ of system (41).

types, and in the use of other regularization methods, without applying models, for example, the residual method. The numerical implementation of the algorithm requires a considerable time, so it is advisable to involve parallel technologies.

\section{Acknowledgments}

This work was supported by the Ural Mathematical Center and the Ural Federal University.

\section{References}

[1] A.N. Tikhonov, V.Y. Arsenin, Solution of Ill-Posed Problems, in: Scripta Series in Mathematics, John Wiley, New York, 1977.

[2] S.I. Kabanikhin, Inverse and Ill-Posed Problems: Theory and Application, in: Inverse and Ill-Posed Problems Series, vol. 55, De Gruyter, Berlin, 2011.

[3] H.T. Banks, K. Kunish, Estimation Techniques for Distributed Parameter Systems, Systems \& Control: Foundations \& Applications, Birkhäuser, Boston, 1998, http://dx.doi.org/10.1007/978-1-4612-3700-6.

[4] A.V. Kryazhimskii, Y.S. Osipov, On positional calculation of $\Omega$-normal control in dynamical system, Probl. Control Inf. Theory 13 (6) (1984) 425-436, http://dx.doi.org/10.1134/S0965542516010139.

[5] Y.S. Osipov, A.V. Kryazhimskii, Inverse Problems for Ordinary Differential Equations: Dynamical Solutions, Gordon and Breach, Basel, 1995.

[6] V.I. Maksimov, Dynamical Inverse Problems of Distributed Systems, in: Inverse and Ill-Posed Problems Series, vol. 37, VSP, Zeist, 2002.

[7] Y.S. Osipov, A.V. Kryazhimskii, V.I. Maksimov, Dynamic Reconstruction Methods for Inputs of Controlled Systems, Ural. Otd. Ross. Akad. Nauk, Yekaterinburg, 2011 (in Russian).

[8] V.I. Maksimov, On dynamical reconstruction of an input in a linear system under measuring a part of coordinates, J. Inverse Ill-Posed Probl. 26 (4) (2018) 395-410, http://dx.doi.org/10.1515/jiip-2017-0118.

[9] V.I. Maksimov, Reconstruction of disturbances in a nonlinear system from measurements of some of the state-vector coordinates, Comput. Math. Math. Phys. 59 (2019) 1771-1780, http://dx.doi.org/10.1134/S0965542519110095.

[10] V.L. Rozenberg, On a problem of dynamic reconstruction under incomplete information, Trudy Inst. Mat. Mek. UrO RAN 25 (1) (2019) $207-218$, http://dx.doi.org/10.21538/0134-4889-2019-25-1-207-218. 
[11] K.J. Keesman, V.I. Maksimov, On feedback identification of unknown characteristics: a bioreactor case study, Internat. J. Control 81 (1) (2008) 134-145, http://dx.doi.org/10.1080/00207170701385843.

[12] F. Fagnani, L. Pandolfi, A singular perturbation approach to a recursive deconvolution problem, SIAM J. Control Optim. 40 (5) (2002) 1384-1405 http://dx.doi.org/10.1137/S0363012900368259.

[13] L. Pandolfi, Adaptive recursive deconvolution and adaptive noise cancellation, Internat. J. Control 80 (3) (2007) 403-415, http://dx.doi.org/10. 1080/00207170601042346.

[14] P.G. Surkov, Dynamic right-hand side reconstruction problem for a system of fractional differential equations, Differential Equations 55 (6) (2019) 849-858, http://dx.doi.org/10.1134/S0012266119060120.

[15] S.G. Samko, A.A. Kilbas, O.I. Marichev, Fractional Integrals and Derivatives: Theory and Applications, Gordon and Breach, Yveron, Switzerland, 1993

[16] A. Carpinteri, F. Mainardi, Fractals and Fractional Calculus in Continuum Mechanics, CISM International Centre for Mechanical Sciences, Springer Verlag, Vienna, 1997, http://dx.doi.org/10.1007/978-3-7091-2664-6.

[17] A.A. Kilbas, H.M. Srivastava, J.J. Trujillo, Theory and Applications of Fractional Differential Equations, in: North-Holland Mathematics Studies, vol. 204, Elsevier Science, New York, 2006 (Book 204).

[18] J. Sabatier, O.P. Agrawal, J.A. Tenreiro-Machado, Advances in Fractional Calculus: Theoretical Developments and Applications in Physics and Engineering, Springer Netherlands, Dordrecht, 2007, http://dx.doi.org/10.1007/978-1-4020-6042-7.

[19] K. Diethelm, The Analysis of Fractional Differential Equations, in: Lecture Notes in Mathematics, vol. 2004, Springer-Verlag, Berlin, Heidelberg, 2010, http://dx.doi.org/10.1007/978-3-642-14574-2.

[20] C. Li, F. Zeng, Numerical Methods for Fractional Calculus, in: Numerical Analysis and Scientific Computing Series, Chapman \& Hall/CRC, Vienna, 2015

[21] P. Agarwal, D. Baleanu, Y. Chen, S. Momani, J.A.T. Machado (Eds.), Fractional Calculus: ICFDA 2018, Amman, Jordan, July 16-18, 2019, http://dx.doi.org/10.1007/978-981-15-0430-3.

[22] N. Heymans, Fractional calculus description of non-linear viscoelastic behaviour of polymers, Nonlinear Dyn. 38 (2004) 221-231, http: //dx.doi.org/10.1007/s11071-004-3757-5.

[23] I. Matychyn, V. Onyshchenko, On time-optimal control of fractional-order systems, J. Comput. Appl. Math. 339 (2018) 245-257, http: //dx.doi.org/10.1016/j.cam.2017.10.016.

[24] L. Peng, Y. Zhou, A. Debbouche, Approximation techniques of optimal control problems for fractional dynamic systems in separable Hilbert spaces, Chaos Solitons Fractals 118 (2019) 234-241, http://dx.doi.org/10.1016/j.chaos.2018.11.025.

[25] C.D. Constantinescu, J.M. Ramirez, W.R. Zhu, An application of fractional differential equations to risk theory, Finance Stoch. 23 (4) (2019) 1001-1024, http://dx.doi.org/10.1007/s00780-019-00400-8.

[26] C.M.A. Pinto, A.R.M. Carvalho, A latency fractional order model for hiv dynamics, J. Comput. Appl. Math. 312 (2017) 240-256, http://dx.doi.org 10.1016/j.cam.2016.05.019.

[27] J. Manimaran, L. Shangerganesh, A. Debbouche, V. Antonov, Numerical solutions for time-fractional cancer invasion system with nonlocal diffusion, Front. Phys. 7 (2019) http://dx.doi.org/10.3389/fphy.2019.00093, article 93.

[28] J.-D. Gabano, T. Poinot, Fractional modeling and identification of thermal systems, Signal Process. 91 (3) (2011) 531-541, http://dx.doi.org/10. 1016/j.sigpro.2010.02.005.

[29] B. Wu, S. Wu, Existence and uniqueness of an inverse source problem for a fractional integrodifferential equation, Comput. Math. Appl. 68 (10) (2014) 1123-1136, http://dx.doi.org/10.1016/j.camwa.2014.08.014.

[30] I.I. Vrabie, Compactness Methods for Nonlinear Evolutions, Monographs and Surveys in Pure and Applied Mathematics, John Wiley \& Sons, New York, 1987.

[31] M.P. Aghababa, Fractional modeling and control of a complex nonlinear energy supply-demand system, Complexity 20 (6) (2015) 74-86, http://dx.doi.org/10.1002/cplx.21533.

[32] S. Ullah, M.A. Khan, M. Farooq, A fractional model for the dynamics of TB virus, Chaos Solitons Fractals 116 (2018) 63-71, http://dx.doi.org/ 10.1016/j.chaos.2018.09.001.

[33] M.I. Gomoyunov, Fractional derivatives of convex Lyapunov functions and control problems in fractional order systems, Fract. Calc. Appl. Anal. 21 (5) (2018') 1238-1261, http://dx.doi.org/10.1515/fca-2018-0066.

[34] Y. Li, Y. Chen, I. Podlubny, Stability of fractional-order nonlinear dynamic systems: Lyapunov direct method and generalized Mittag-Leffler stability, Comput. Math. Appl. 59 (2010) 1810-1821, http://dx.doi.org/10.1016/j.camwa.2009.08.019.

[35] S. Momani, Z. Odibat, Numerical approach to differential equations of fractional order, J. Comput. Appl. Math. 207 (1) (2007) 96-110, http://dx.doi.org/10.1016/j.cam.2006.07.015. 